\title{
Mental illness in the Western Cape Province, South Africa: A review of the burden of disease and healthcare interventions
}

\author{
N Jacob, MB ChB, FCPHM, MMed (Public Health Medicine); D Coetzee, BA, MB BCh, DTM\&H, FFCH (SA), MS (Epidemiology) \\ Western Cape Government: Health, Cape Town; and School of Public Health and Family Medicine, Faculty of Health Sciences, University of \\ Cape Town, South Africa
}

Corresponding author: N Jacob (nisha.jacob@uct.ac.za)

\begin{abstract}
Neuropsychiatric disorders were ranked third as contributors to disability-adjusted life-years in South Africa (SA). Despite this high morbidity, mental health is often overlooked on the public health agenda. This article reviews evidence on the burden of mental illness in the Western Cape Province of SA, as well as current provincial interventions to decrease the burden of mental illness. Available evidence supports the need for improved integration of mental health services in primary healthcare and strengthening of community services. Challenges include a lack of capacity due to staff shortages and inadequate availability and allocation of resources. Evidence from large epidemiological studies to quantify the burden of disease as well as cost-effectiveness studies of interventions are required to successfully plan and implement interventions. Similar reviews may provide a national overview of mental health issues as well as allow improvement through better understanding of research and best practices in various provinces.
\end{abstract}

S Afr Med J 2018;108(3):176-180. DOI:10.7196/SAMJ.2018.v108i3.12904

Mental health has a major impact on health at both individual and population level. The recent national tragedy resulting in the death of more than 94 mental healthcare users in Gauteng Province, South Africa (SA), has cast a spotlight on public mental healthcare and associated systemic inadequacies. Based on the revised national disability-adjusted life-year (DALY) estimates from the SA National Burden of Disease Study, ${ }^{[1]}$ neuropsychiatric disorders were ranked third, after HIV/AIDS and other infectious diseases, as contributors to the burden of disease, surpassing other non-communicable diseases (NCDs). ${ }^{[1]}$ Globally, there is an increasing recognition of mental illness.

In 2007, the Western Cape Provincial Department of Health embarked on the Burden of Disease Reduction Project. ${ }^{[2]}$ A Mental Health Workgroup (MHW) was established to make recommendations on interventions to reduce the burden of mental illness in the province. Although a large focus of the report was on interventions addressing upstream determinants of health, downstream service interventions were also proposed. In this review, we briefly assess evidence regarding the current burden of mental illness in the Western Cape as well as service-related interventions that have been implemented following recommendations from the $2007 \mathrm{MHW}$.

\section{Methods}

Evidence for this review was obtained from a comprehensive literature review and interviews with 10 key mental health experts in the province, including programme managers, psychiatrists and members of the provincial Mental Health Policy Working Group. The main search engines used were PubMed and Google Scholar. Key search terms included 'mental health' AND 'South Africa' AND 'Western Cape' AND 'prevalence mental illness' AND 'mental health interventions'. All relevant articles published in English from 1990 to 2015 were included. Reference lists were scanned further. Unpublished literature, including provincial reports, was sourced from key stakeholders. Only key references are presented in this article.

\section{Review findings \\ The burden of mental illness categories, viz.: \\ 1. Prevalence or incidence of mental illness \\ 2. Risk factors for mental illness \\ 3. Consequences in terms of DALYs \\ 4. Social and economic costs.}

The burden of mental illness can be assessed within four major

In the Western Cape, there are currently limited reliable data on the burden of mental illness. The only routine mental health indicators at primary healthcare (PHC) services are the number of mental health clients aged $<18$ years and $\geq 18$ years. These data are obtained mainly from mental health nurses at primary-level facilities and are likely to capture only individuals with serious mental illness who are referred to mental health nurses. Patients with more common, less severe mental illness are not likely to be included in mental health service data. There are also no indicators on substance use. Data on psychiatric admissions and 90-day readmission rates are collected at secondary and tertiary levels of care. There are limited epidemiological studies quantifying the burden of mental illness in the Western Cape and in SA at large. The available data are summarised below.

\section{Prevalence and incidence}

Adults

The South African Stress and Health Survey (SASH) conducted in 2004 remains the main source of mental health prevalence data for SA. ${ }^{[3]}$ This study was the first large-scale population-based study of common mental disorders in SA. ${ }^{[3]}$ It showed that the 12-month prevalence of common mental disorders among SA adults was $16.5 \%$, that the lifetime prevalence for any disorder was $30.3 \%$, and that the Western Cape had the highest 12-month and lifetime prevalence in SA (39.4\%). ${ }^{[3]}$ In the Western Cape, the prevalence of anxiety disorders was $18.9 \%$, that of mood disorders $13.7 \%$ and that 
of substance use disorders $20.6 \%{ }^{[3]}$ Confidence intervals for these proportions were not made available.

When stratified by province, the sample sizes for the SASH study were small and subject to random error. Another limitation of the study was the exclusion of individuals with psychotic disorders, which meant that the overall lifetime prevalence of mental disorders was underestimated. Although a relatively small proportion of the population is affected by these disorders, they have a major impact on health services.

Suicide data are often used as a proxy for mental illness burden, since $\sim 90 \%$ of people who commit suicide have a psychiatric disorder at the time of their death. ${ }^{[2]}$ Suicide accounted for $\sim 11 \%$ of all unnatural deaths in 2012, ${ }^{[4]}$ although suicide figures in isolation are a gross underestimation of underlying mental disorders. ${ }^{[2]}$

The 2007 MHW recommended that mortality data on injuries may be a more appropriate proxy measure for the mental health burden than suicide data. ${ }^{[2]}$ In 2012, interpersonal violence was among the top five causes of mortality in all Western Cape districts and was the second most common cause of years of life lost among men. ${ }^{[4]}$ These data had remained fairly consistent over the preceding 3 years.

The prevalence of mental disorders at primary-level facilities is likely to be underestimated, as the SASH study revealed that only $25 \%$ of participants meeting the criteria for a mental disorder sought treatment, owing to a low perceived need for mental healthcare and poor mental health literacy. ${ }^{[3,5]}$ Mental health service data underestimate the burden as a result of underdiagnosis and poor access to mental healthcare due to structural and attitudinal barriers. ${ }^{[5]}$

\section{Children and adolescents}

The SASH study excluded children and adolescents, and data on the prevalence of mental illness in this group are lacking.

Kleintjes et al. ${ }^{[6]}$ estimated that the unadjusted prevalence of any mental disorder among children and adolescents in the Western Cape was $17 \% .{ }^{[6]}$ The adjusted prevalence estimate for generalised anxiety disorder was $11 \%$, that for post-traumatic stress disorder (PTSD) $8 \%$ and that for major depressive disorder and dysthymia $8 \%{ }^{[6]}$

In $2011,11 \%$ of non-natural deaths in the 10 - 14-year age group and $10 \%$ in the 15 - 19-year age group were due to suicide. ${ }^{[4]}$ Although some suicides may be impulsive, these figures point to the need for improved mental health among adolescents.

Morojele et al. ${ }^{[7]}$ showed that $41.4 \%$ of grade 8 - 10 learners in Western Cape schools were at medium risk and $14.9 \%$ at high risk for mental health problems. Female learners were more likely to be at high risk. ${ }^{[7]}$

\section{Risk factors}

Pertinent downstream risk factors for mental illness include comorbidities such as HIV as well as NCD, disorders during the perinatal period, substance abuse and experience of trauma. ${ }^{[2,8]}$

\section{HIV}

A synergistic interaction exists between HIV and mental illness, with depression, anxiety, PTSD and alcohol abuse being the most prevalent disorders among people living with HIV ${ }^{[9]}$ Depression, anxiety and stressful life events are associated with a worsened course of illness among those with HIV. ${ }^{[9]}$ Furthermore, mental disorders are associated with poor adherence to antiretroviral treatment. ${ }^{[0]}$ Mental illness is also a risk factor for HIV infection owing to impaired judgement and insight and associated high-risk behaviour.

Few studies have been conducted on the burden of mental illness among individuals with HIV. Myer et al. ${ }^{[9]}$ showed that $19 \%$ of those attending routine HIV follow-up care in Cape Town had mental disorders, including depression, PTSD and alcohol dependence.

\section{NCDs}

A similar synergistic interaction exists between NCDs and mental illness. ${ }^{[2]}$ Mental disorders and other NCDs have similar risk factors, e.g. alcohol abuse is associated with depression and anxiety disorders and is also a risk factor for cardiovascular disease. ${ }^{[2]}$ Depression and anxiety both increase the risk for hypertension, and depression is an independent risk factor for stroke and type 2 diabetes. ${ }^{[2]} \mathrm{A}$ high level of comorbidity between NCDs and mental illness is expected, although data in the Western Cape are largely limited to facility data, so are likely to be underestimated.

\section{Disorders in women}

Numerous studies show that women are more likely to have depression, anxiety disorders and severe mental disorders. ${ }^{[3,10]}$ Women have an increased risk of mental health disorders during the perinatal period, and these disorders are associated with increased maternal morbidity and mortality, and adverse child health outcomes. ${ }^{[2,10]}$ Studies in the Western Cape show a high prevalence of various common mental disorders among pregnant women. ${ }^{[10]}$ Of major concern was the large proportion of pregnant women with multiple risk factors for adverse perinatal outcomes, including depression and alcohol use. ${ }^{[10]}$

\section{Substance abuse and experience of trauma}

Alcohol and other psychoactive substances are widely recognised risk factors for mental illness and injuries. ${ }^{[3]}$ Substance abuse contributes to a large proportion of non-natural deaths in the province. ${ }^{[4]}$ The extensive use of methamphetamine (commonly known as 'tik'), particularly among adolescents, is of major concern in the Western Cape. ${ }^{[7]}$ Studies showed a marked increase in admissions and re-admissions due to methamphetamine abuse. ${ }^{[7,8]}$ Morojele et al..$^{[7]}$ found that $>60 \%$ of adolescent learners reported having witnessed a community member being beaten, $40 \%$ had observed a stabbing and $12 \%$ had witnessed forced sexual intercourse in a 12 -month period. ${ }^{[7]}$ These factors further contribute to mental health problems, including PTSD, anxiety disorders and mood disorders.

\section{Consequences of mental illness in terms of DALYs}

DALYs are valuable indicators to quantify the burden of mental illness, as both morbidity and mortality are included. ${ }^{[1]}$ This is particularly useful when mortality rates are low. ${ }^{[1]}$ In 2005, the World Health Organization attributed $31.7 \%$ of all years lived with disability to neuropsychiatric conditions, including depression, alcohol use disorders, schizophrenia, bipolar mood disorders and dementia. ${ }^{[1]}$

In SA in 2000, neuropsychiatric disorders ranked third in DALYs. ${ }^{[1]}$ It is challenging to derive accurate DALY estimations, as there are intensive data requirements and disability weights used in SA for DALY estimations are not context specific. ${ }^{[1,1]}$ In order to develop more accurate burden measures, experts recommend that disability weights should be empirically assessed in SA rather than based on data from different countries. ${ }^{[1,11]}$

\section{Societal/economic costs}

Limited evidence is available on the societal and economic costs of mental illness in the Western Cape. Although DALYs provide some information on societal costs, they do not include indirect costs such as caregiver burnout or the negative impact on the family.

Direct economic costs include the cost of medical care and services, whereas indirect costs include lost productivity, unemployment and 
disability benefits. Indirect costs tend to outweigh direct costs in most studies. ${ }^{[11]}$

In the private sector, medication costs are notably high; however, no data are available for the public sector. ${ }^{[11]}$ Staff expenditure also contributes significantly to the direct costs of mental healthcare. ${ }^{[11]}$

Lund et al. ${ }^{[1]]}$ estimated the indirect costs of mental illness through lost earnings at USD4 798 (ZAR54 121) per adult with major depression and anxiety disorders per annum. Projections of the total annual cost of these disorders in lost earnings were USD3.6 billion in 2003. ${ }^{[11]}$ This indicates that mental illness has a significant economic impact.

\section{Mental health interventions in the health services}

Mental health interventions may target individuals or populations, and span all levels of prevention, viz. primordial, primary, secondary and tertiary prevention.

The 2007 Burden of Disease Reduction report ${ }^{[2]}$ detailed core intervention areas for mental illness, viz.:

- Multiple deprivation. Interventions aimed at improving quality of living as well as providing employment and education.

- Substance abuse. Population-level interventions for substance abuse such as the enforcement of regulatory legislation, increased costs of alcohol, and reduced availability of alcohol.

- Mental health services. Interventions that target health facilities across all levels of care, e.g. integration of mental health services in general medical services, community programmes, dedicated psychiatric services, etc.

- Trauma. Mental health intervention at trauma facilities and through occupational groups that work with trauma victims to improve treatment of post-traumatic states that may precipitate or exacerbate mental illness.

- Preschool education. The development of quality early-childhood development programmes to reduce the burden of mental illness.

- Recreation. Involvement in recreational activities from early childhood is considered essential in the prevention and management of negative life events.

The majority of these interventions address upstream factors associated with mental health, requiring an intersectoral approach in the development of primordial and primary prevention strategies. This article primarily focuses on health service-related interventions in the Western Cape, which are mainly directed towards secondary and tertiary prevention strategies.

Although the South African Mental Health Care Act ${ }^{[12]}$ mandates the integration of mental health services into PHC, many barriers exist globally, as listed in Table 1.

\section{Recommended service-related interventions}

Various service-related interventions were recommended by the 2007 MHW. More recently, Petersen et al..$^{[8]}$ conducted a systematic review of research conducted in mental health services in South Africa from 2000 to 2010 and identified key recommendations, many of which overlap with the abovementioned interventions.

Table 2 categorises the main recommended interventions for the Western Cape from the available literature. Since economic evaluations of these interventions are limited, they have been assessed qualitatively based on the key requirements for successful implementation, i.e. training, human resources and dedicated allocation of resources (denoted with ${ }^{*}$ ). The final column shows current interventions in the province, which are explained in further detail below.

Available evidence as well as legislation strongly supports integrated primary mental healthcare packages and strengthening of community-based services. ${ }^{[8]}$ Horizontal integration is particularly suited to the SA setting, given the growing prevalence of comorbidities ${ }^{[8]}$ The SASH study showed that most adults utilised general medical services rather than specific mental health services for mental health problems. ${ }^{[3]}$ Integrated interventions at primary-level facilities could improve coverage for those at risk for mental disorders who access healthcare services, thereby increasing the impact and cost-effectiveness of interventions as well as improving overall health outcomes by increasing adherence for all chronic diseases. Strengthening of community-based services is well supported by the literature. ${ }^{[8,13]}$

Task shifting is one of the many methods recommended to integrate mental health within primary-level care. ${ }^{[8]}$ Task shifting involves the provision of mental health at community and clinic levels using lower levels of healthcare workers. Primary-level services are strengthened by training current medical professionals, community health workers (CHWs) and counsellors in mental health. This approach is less costly than alternative staffing models that rely on specialist personnel. ${ }^{[8]}$ Petersen et al. ${ }^{[8]}$ motivated for the addition of mental health counsellors with a 4-year bachelor of psychology degree to the staff at PHC clinics. ${ }^{[8]}$ The roles of such counsellors would include support to community mental health workers, screening and referral of severe cases and counselling services for PTSD ${ }^{[8]} \mathrm{A}$ number of interventions in the Western Cape require additional non-specialist staff. Although these innovations are more costeffective than the use of specialist personnel, the addition of a cadre of lower-level mental health workers would require considerable funding. Training and task-shifting using existing personnel may be the least costly option in resource-constrained settings. A concern is the over-burdening of already pressured staff, leading to poor uptake of task-shifting approaches.

Table 1. Barriers to integration of mental health services into PHC

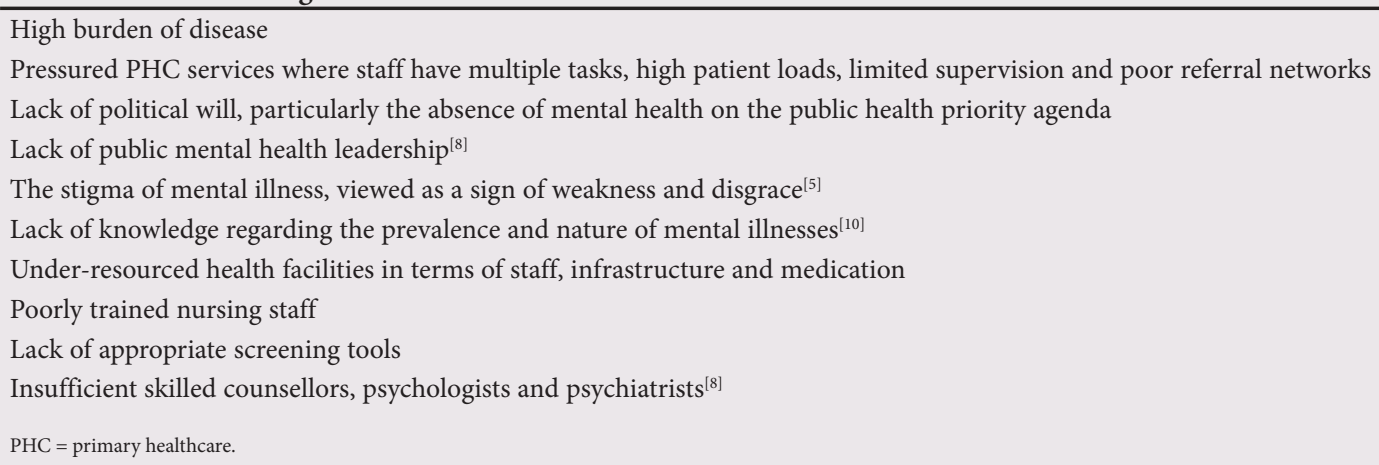


Table 2. Recommended interventions to enhance mental health services

\begin{tabular}{|c|c|c|c|c|}
\hline Recommended interventions & Training & $\begin{array}{l}\text { Human } \\
\text { resources }\end{array}$ & $\begin{array}{l}\text { Resource } \\
\text { allocation }\end{array}$ & $\begin{array}{l}\text { Current } \\
\text { intervention }\end{array}$ \\
\hline \multicolumn{5}{|l|}{ General interventions } \\
\hline \multicolumn{5}{|l|}{$\begin{array}{l}\text { A. Integration into general medical services at all levels of care, } \\
\text { particularly in the following services: } \\
\text { - Antenatal and postnatal } \\
\text { - HIV/AIDS } \\
\text { - Trauma } \\
\text { - NCD }\end{array}$} \\
\hline $\begin{array}{l}\text { Task-shifting. Training of general medical staff (including nurses and } \\
\text { doctors) to deliver basic mental health services including screening } \\
\text { and interventions for substance abuse and dependences }\end{array}$ & * & & * & PACK \\
\hline $\begin{array}{l}\text { Task-shifting. Employment of new cadres of low-level healthcare } \\
\text { workers }\end{array}$ & * & * & & $\begin{array}{l}\text { PMHP; } \\
\text { counselling in } \\
\text { EC centres }\end{array}$ \\
\hline \multicolumn{5}{|l|}{ Ensure appropriate referral networks } \\
\hline \multicolumn{5}{|l|}{ B. Development of community-based mental health services } \\
\hline \multirow{2}{*}{$\begin{array}{l}\text { Improve community-based rehabilitation and care facilities } \\
\text { Task-shifting. Training of CCWs in mental health screening, } \\
\text { counselling and adherence support }\end{array}$} & & & * & \\
\hline & * & & * & $\begin{array}{l}\text { DoH/NPO } \\
\text { partnerships } \\
\text { for CCWs }\end{array}$ \\
\hline Mental health awareness initiatives in the community & & & * & NGOs \\
\hline \multirow{2}{*}{$\begin{array}{l}\text { C. Improve infrastructure at all levels of care } \\
\text { D. Ensure that psychotropic medication is universally available at } \\
\text { primary-level services }\end{array}$} & & & * & \\
\hline & & & * & \\
\hline \multirow{2}{*}{$\begin{array}{l}\text { E. Promote culturally appropriate care } \\
\text { F. Services provided in African languages, particularly isiXhosa and } \\
\text { Afrikaans in the Western Cape }\end{array}$} & * & & & \\
\hline & * & & & \\
\hline \multicolumn{5}{|l|}{ Specialist interventions } \\
\hline \multirow{2}{*}{$\begin{array}{l}\text { A. Employ dedicated mental health professionals at district hospitals } \\
\text { B. Make provision for specialist and subspecialist psychiatry posts at } \\
\text { all levels of care }\end{array}$} & & * & & \\
\hline & & * & * & \\
\hline \multirow{2}{*}{$\begin{array}{l}\text { C. Provide adequate substance dependence treatment services } \\
\text { D. Improve integration between mental health and substance services }\end{array}$} & & & * & \\
\hline & * & & * & \\
\hline
\end{tabular}

$\mathrm{NCD}=$ non-communicable disease; PACK = Practical Approach to Care Kit; PMHP = Perinatal Mental Health Project; EC = emergency centre; DoH = Department of Health; NPO = non-profit

${ }^{*}$ Key requirement for implementation of the interventions listed in the first column.
* $\mathrm{CCW}$.

\section{Integrated mental healthcare in the Western Cape}

The Mental Health Policy Working Group is currently developing the Mental Health Policy Framework for the province, using a life-course approach.

Service-related interventions are being developed, piloted and rolled out in the province. The main focus is the integration of mental health in PHC as well as the strengthening of community-based services.

The Practical Approach to Care Kit (PACK), adopted by the Western Cape, is a comprehensive clinical practice guideline for use by clinicians to diagnose and manage common conditions at primary level in an integrated manner, including mental illness. ${ }^{[14]}$ The mental health module includes diagnosis and management guidelines for voluntary and involuntary admission procedures, depression, anxiety, substance abuse, psychosis and dementia. ${ }^{[14]}$

The Perinatal Mental Health Project (PMHP) is another primarylevel intervention in selected facilities, providing psychiatric screening, counselling and treatment services to pregnant women. ${ }^{[1]}$ Women are screened at their first antenatal visit to identify those at risk, who are then referred to an on-site counsellor for further management and referral if necessary. This validated screening tool may be used by lay workers, CHWs, nurses and other healthcare workers.
Interventions targeting those at risk, such as pregnant women, promote mental wellness by increasing the adaptive capabilities of women, improving agency and productivity as well as general living conditions, ${ }^{[11]}$ thus reducing expenditure on health.

The Substance Use and Trauma Intervention (STRIVE) study, conducted in 2012/2013, describes another intervention using the integrated care approach to decrease substance abuse and its negative effects among patients attending emergency centres (ECs) in the Western Cape. ${ }^{[15]}$ The cost-effectiveness of interventions delivered by trained peer counsellors in the emergency setting was evaluated. ${ }^{[15]}$ The intervention reduced substance abuse and depressive symptoms among EC patients. ${ }^{[15]}$ The cost of employing peer counsellors was approximately five times less than that of appointing a trained clinical psychologist to screen and deliver the intervention. Again, although the intervention was cost-effective, the feasibility of appointing new cadres of staff at various facilities in the province needs further exploration.

\section{Home and community-based care services in the Western Cape}

Strengthening of community-based services is an important intervention described in the literature. Community-based services 
are delivered by CHWs or community care workers (CCWs), community rehabilitation workers and lay counsellors.

In the Western Cape, community-based services are provided through contracts with non-profit organisations (NPOs) employing about 3500 CCWs, supported and supervised by NPO-appointed professional nurses. While initial community-based services were specialised, e.g. tuberculosis and HIV home-based care, the services are becoming more integrated and comprehensive. CCWs can play an important role in the prevention of mental illness through health promotion, screening and adherence support.

A recent provincial discussion document (unpublished) outlines the framework for home- and community-based care services in the Western Cape. Core competencies for CCWs include knowledge on mental health and other NCDs, as well as counselling skills. There is, however, variability in the training and functions of CCWs from different NPOs, although the current service package attempts to standardise the services provided. The National Department of Health is developing a standardised, comprehensive training qualification for CHWs, but the proposed training qualification incorporates very basic mental health training. Both the PACK and PMHP propose that CCWs receive adequate training and support to screen, refer and provide basic care for those in psychological distress as part of their routine tasks.

An area of debate is whether CCWs should be trained as generalists, who incorporate mental health into their routine tasks, or as specialists with an exclusive focus on mental health issues. Given the resource constraints, a generalist approach appears more feasible, but the expectation that multiple health issues should be covered in routine CCW tasks may be overwhelming and important aspects of the health encounter may be neglected. Studies comparing the cost-effectiveness of generalist and specialist CCWs are required to provide more robust evidence for such debates.

\section{Other interventions in the Western Cape}

Community follow-up of discharged patients by hospital teams known as Assertive Community Teams was established in the Western Cape in 2007. There is evidence that this type of intervention may be particularly effective where community mental health services are inadequate. ${ }^{[16]}$ Given the high cost and resource requirements for this intervention, improved community mental health services may be more cost-effective.

Other provincial mental health interventions include nongovernmental organisation-led initiatives as well as research-driven interventions including the Programme for Improving Mental Health Care (PRIME), the Emerging Mental Health Systems in Low- and Middle-income Countries (EMERALD) project, the Africa Focus on Intervention Research for Mental Health (AFFIRM) project, and Project MIND. Feedback from such research should be presented to all stakeholders in order to strengthen policy development.

\section{Recommendations}

Evidence on the burden of mental disease in the Western Cape is limited, and largely outdated. A population-based epidemiological study to estimate the burden of mental disease is recommended. Current mental health service indicators are poorly reflective of the true burden of disease, and there is a need for more valid indicators.

The main areas of intervention that may be feasible and effective are: - Strengthening community-based services through training of CCWs
- Integration of mental health services through task-shifting of existing staff at PHC, antenatal, postnatal, trauma, HIV and NCD services

- Consideration of employment of a new cadre of mental health staff at subdistrict or facility level who may function at facility and community level.

Given the limited evidence available locally and nationally, there is a need for economic evaluations of mental health interventions.

\section{Conclusion}

Mental illness contributes significantly to the burden of disease in the Western Cape. Although this review focuses on one province, many issues are pertinent to any under-resourced setting. A concerted effort by policy makers is required to ensure that appropriate information on mental illness is captured and interventions are based on sound evidence. Although many promising interventions are underway, there is a need to bolster mental health services at all levels of healthcare. An evidence-based intersectoral approach is required to optimise mental health in the population and truly achieve health for all.

Acknowledgements. Juliet Evans, Crick Lund, Peter Milligan and Marinda Roelofse.

Author contributions. NJ conducted the review. DC made significant intellectual contributions.

Funding. Western Cape Government: Health.

Conflicts of interest. None.

1. Bradshaw D, Norman R, Schneider M. A clarion call for action based on refined DALY estimates for South Africa. S Afr Med J 2007;97(6):438-440.

2. Corrigall J, Ward C, Stinson K, et al. Decreasing the burden of mental illness: Final report 2007 (Volume 4 of 7). In: Myers JE, Naledi NT, eds. Western Cape Burden of Disease Reduction Project. University of Cape Town on behalf of the Provincial Department of Health, 2007. https://www.researchgate.net/ publication/242703525_Decreasing_the_Burden_of_Mental_Illness (accessed 5 February 2019).

3. Herman AA, Stein DJ, Seedat S, Heeringa SG, Moomal H, Williams DR. The South African Stress and Health (SASH) study: 12-month and lifetime prevalence of common mental disorders. S Afr Med J 2009;99(5):339-344.

4. Groenewald P, Evans J, Morden E, et al. Western Cape Mortality Profile 2012. Cape Town: South African Medical Research Council, 2015.

5. Bruwer B, Sorsdahl K, Harrison J, Stein DJ, Williams D, Seedat S. Barriers to mental health care Bruwer B, Sorsdahl K, Harrison J, Stein DJ, Williams D, Seedat S. Barriers to mental health care
and predictors of treatment dropout in the South African Stress and Health Study. Psychiatric Serv and predictors of treatment dropout in the South African Stress

6. Kleinties S, Flisher A, Fick M, et al. The prevalence of mental disorders among children, adolescents Kleintjes S, Flisher A, Fick M, et al. The prevalence of mental disorders among children, adolescents
and adults in the Western Cape, South Africa. S Afr Psychiatry Rev 2006;9(3):157-160. https://doi. and adults in the Western $\mathrm{C}$,
org/10.4314/ajpsy.v9i3.30217

org/10.4314/ajpsy.v9i3.30217
7. Morojele N, Myers B, Townsend L, et al. Survey on Substance Use, Risk Behaviour and Mental Health Morojele N, Myers B, Townsend L, et al. Survey on Substance Use, Risk Behaviour and Mental Health
Among Grade 8 - 10 Learners in Western Cape Provincial Schools, 2011. Cape Town: South African Medical Research Council, 2013.

8. Petersen I, Lund C. Mental health service delivery in South Africa from 2000 to 2010: One step forward, one step back. S Afr Med J 2011;101(10):751-757.

9. Myer L, Smit J, Roux LL, Parker S, Stein DJ, Seedat S. Common mental disorders among HIV-infected individuals in South Africa: Prevalence, predictors, and validation of brief psychiatric rating scales. AIDS Patient Care STDS 2008;22(2):147-158. https://doi.org/10.1089/apc.2007.0102

10. Meintjes I, Field S, van Heyningen T, Honikman S. Creating capabilities through maternal mental health interventions: A case study at Hanover Park, Cape Town. J Int Dev 2015;27(2):234-250. https:// doi.org/10.1002/jid.3063

11. Lund C, Myer L, Stein DJ, Williams DR, Flisher AJ. Mental illness and lost income among adult South Africans. Soc Psychiatry Psychiatr Epidemiol 2013;48(5):845-851. https://doi.org/10.1007/s00127 012-0587-5

2. South Africa. Mental Health Care Act No. 17 of 2002. http://www.gov.za/sites/www.gov.za/files/a17-02 pdf (accessed 9 February 2017)

13. Naledi T, Barron P, Schneider H. Primary health care in SA since 1994 and implications of the new vision for PHC re-engineering. In: South African Health Review 2011. Durban: Health Systems Trust, 2011:17-28.

14. Practical Approach to Care Kit. Knowledge Translation Unit, University of Cape Town Lung Institute. http://knowledgetranslation.co.za/programmes (accessed 5 May 2015).

15. Dwommoh RAK. Brief Interventions to Address Substance Use in Emergency Departments in the Western Cape: A Cost-effectiveness Analysis. Cape Town: University of Cape Town, 2014. https:// open.uct.ac.za/handle/11427/6031 (accessed 12 April 2015)

6. Botha UA, Koen L, Galal U, Jordaan E, Niehaus DJ. The rise of assertive community interventions in South Africa: A randomized control trial assessing the impact of a modified assertive intervention on readmission rates; A three year follow-up. BMC Psychiatry 2014;14:56. https://doi.org/10.1186/1471-
244x-14-56

Accepted 20 November 2017. 